\title{
L-ANALYTIC MAPPINGS IN THE DISK ALGEBRA
}

\author{
H. E. WARREN
}

\begin{abstract}
It is shown that two classes of function transformations coincide when the transformations take place within the disk algebra. The first class is that of the $L$-analytic mappings. These are the ones given locally by power series: $f \rightarrow \sum g_{n}\left(f-f_{0}\right)^{n}$. The second class is that of locally pointwise mappings. A mapping $f \rightarrow \Phi[f]$ is pointwise if it has the form $\Phi[f](x)=\Phi^{*}(x, f(x))$. It is a by-product of the disk algebra investigation that if a set $X$ has certain topological properties, then every locally pointwise mapping in $C(X)$ is continuous.
\end{abstract}

In 1943 E. R. Lorch introduced an analytic theory for mappings whose domain and range lie in a commutative Banach algebra with identity [3]. Let $A$ be such an algebra, and let $D$ be an open connected subset of $A$. A mapping $\Phi: D \rightarrow A$ is $L$-analytic, that is analytic in the sense of Lorch if in a neighborhood of each $g \in D$ we have a power series expansion $\Phi[f]=\sum g_{n}(f-g)^{n}$. The series is to converge in the norm of $A$, and the coefficients $g_{n}$ are elements of $A$ (which depend on $g$ ).

To study $L$-analytic mappings it is standard procedure to use a technique similar to the Gelfand transform. Let $M$ be a complex homomorphism of $A$ onto the complex numbers $C$. We say $\Phi: D \rightarrow A$ quotients on $D$ with respect to $M$ if there is an ordinary holomorphic function $\Phi_{M}$ which is defined on $M(D)$ and satisfies $\Phi_{M} \circ M=M \circ \Phi$. Call $\Phi_{M}$ the quotient function of $\Phi$ at $M$. If $\Phi$ quotients on $D$ with respect to every $M$, we say $\Phi$ quotients on $D$. On certain domains, for instance on balls, an $L$ analytic mapping will always quotient. For more on quotient functions see [1]. The fact that a mapping quotient has an important interpretation when $A$ is a "natural" algebra of functions on a space $X$. By "natural" we mean that $A$ determines the topology of $X$ and that every homomorphism of $A$ onto $C$ is given by evaluation at a point of $X$. The terminology is due to Rickart [4].

Let $A$ be a natural algebra of functions on a space $X$, and let $E_{x}$ be the evaluation functional $E_{x}(f)=f(x)$. To say $\Phi: D \rightarrow A$ quotients on $D$ is to say that for each $x \in X$ we have a quotient function $\Phi_{x}$ that satisfies

Received by the editors August 30, 1972.

AMS (MOS) subject classifications (1970). Primary 30A96, 30A98; Secondary 46J15. 
$\Phi_{x} \circ E_{x}=E_{x} \circ \Phi$, that is $\Phi[f](x)=\Phi_{x}(f(x))$ for all $f \in D$. The force of this last equation is that $\Phi$ is a pointwise mapping. In general, a mapping $\Phi: D \rightarrow A$ is pointwise on $D$ if there is an auxiliary function $\Phi^{*}$ such that $\Phi[f](x)=\Phi^{*}(x, f(x))$ for all $f \in D$ and $x \in X$. If $\Phi$ quotients on $D$, then $\Phi$ is pointwise on $D$ with $\Phi^{*}(x, y)=\Phi_{x}(y)$.

In [6] this author studied the possibility of proving a Riemann mapping theorem for the Lorch analytic theory in the case $A=C(X)$. Critical use was made of the fact that an $L$-analytic mapping defined on the unit ball of $C(X)$ must be pointwise. In trying to extend the results of [6] to the case when $A$ is the disk algebra it came as a surprise that the pointwise property can in turn imply $L$-analyticity.

THEOREM 1. If $\Phi$ is a pointwise mapping from an open ball $B$ in the disk algebra back into the disk algebra, then $\Phi$ is L-analytic on $B$.

The proof of the theorem will follow a list of notations and three lemmas.

Notations. The letter $C$ will stand for the complex numbers. Put $K=\{z \in C ;|z| \leqq 1\}$ and $T=\{z \in C ;|z|=1\}$. From now on $A$ will denote the disk algebra, the algebra of continuous functions from $K$ to $C$ which are analytic on the interior of $K$. The algebra operations are pointwise addition and multiplication. If $f \in A$, then the norm of $f$ is $\|f\|=$ $\max \{|f(z)| ; z \in K\}$. The open unit ball in $A$ is $U=\{f \in A ;\|f\|<1\}$. Its closure is $\bar{U}=\{f \in A ;\|f\| \leqq 1\}$. We shall think of $C$ as embedded in $A$ by identifying a complex number $z$ with the function on $K$ identically equal to $z$. This embedding is an isometry, that is, $\|z\|=|z|$. Notice that $K \subset \bar{U}$. When we deal with a mapping $\Phi: \bar{U} \rightarrow A$, the image of $z \in K$ under $\Phi$ is $\Phi[z]$. This $\Phi[z]$ is a function; its value at $w \in K$ is $\Phi[z](w)$.

We need some facts about integrals of $A$-valued functions. Given a mapping $\gamma: T \rightarrow A$, let $\gamma[\zeta]$ be the image function of $\zeta \in T$ under $\gamma$. The integral $\int_{T} \gamma[\zeta] d \zeta$ is defined as a limit of Riemann sums in $A$.

LEMMA 1. If $\gamma: T \rightarrow A$ is continuous, then $\int_{T} \gamma[\zeta] d \zeta$ exists and represents a function ir. $A$.

One proves this result just as in the classical case of complex valued functions. It is important to note that if $b=\int_{T} \gamma[\zeta] d \zeta$ then $b(z)=$ $\int_{T} \gamma[\zeta](z) d \zeta$. The reason is that point evaluation is a continuous functional and may therefore be interchanged with the limit of Riemann sums which defines the integral.

Suppose now that $f \in U$ and $\zeta \in T$. Since $\zeta-f$ is a nonvanishing function, we have $(\zeta-f)^{-1} \in A$. In fact $\gamma_{f}[\zeta]=(\zeta-f)^{-1} \gamma[\zeta]$ defines a continuous mapping $\gamma_{f}: T \rightarrow A$, whenever $\gamma: T \rightarrow A$ is continuous. By the lemma above $\int_{T} \gamma_{f}[\zeta] d \zeta$ belongs to $A$. 
LEMMA 2. If $\gamma: T \rightarrow A$ is continuous and

then $\Psi$ is L-analytic on $U$.

$$
\Psi[f]=(2 \pi i)^{-1} \int_{T}(\zeta-f)^{-1} \gamma[\zeta] d \zeta,
$$

Once again the proof follows that of the classical result. See for example [2]. We shall exploit the fact that, roughly speaking, $\Psi$ is the Cauchy integral of the boundary values given by $\gamma$. The next lemma starts the work on pointwise mappings.

Lemma 3. If $\Phi: \bar{U} \rightarrow A$ is a pointwise mapping, then $\Phi$ is continuous.

Proof. We start with two important remarks. First, if $E$ is a subset of the unit circle which is closed and of measure zero and if $g$ is a continuous function on $E$ with $g(E) \subset K$, then $g$ extends to a function in $\bar{U}$. This result is due to Rudin [5]. Second, if $g \in \bar{U}$ then $\Phi[g]$ is continuous on $K$. That is because $\Phi$ maps $\bar{U}$ into $A$.

Let $\Phi[f](z)=\Phi^{*}(z, f(z))$ as in the definition of pointwise mapping. In the present case $z$ and $f(z)$ can both range over $K$. We show first that for any $z_{0} \in T$ the mapping $w \rightarrow \Phi^{*}\left(z_{0}, w\right)$ is continuous on $K$. If this were false there would exist an $\varepsilon>0$ and complex numbers $w_{0}, w_{1}$, etc. in $K$ satisfying $w_{n} \rightarrow w_{0}$ and $\left|\Phi^{*}\left(z_{0}, w_{0}\right)-\Phi^{*}\left(z_{0}, w_{n}\right)\right| \geqq \varepsilon$ for $n \geqq 1$. Consider the $w_{n}$ in their role as constant functions in $\bar{U}$. Since the functions $\Phi\left[w_{n}\right]$ are continuous at $z_{0}$ we can choose $z_{1}, z_{2}$, etc. with the following properties:

(1) $z_{n} \in T$,

(2) $z_{n} \rightarrow z_{0}$,

(3) the $z_{n}$ are distinct from each other and from $z_{0}$, and

(4) $\left|\Phi\left[w_{n}\right]\left(z_{0}\right)-\Phi\left[w_{n}\right]\left(z_{n}\right)\right|<\varepsilon / 2$.

Condition (4) is the same as $\left|\Phi^{*}\left(z_{0}, w_{n}\right)-\Phi^{*}\left(z_{n}, w_{n}\right)\right|<\varepsilon / 2$.

Let $E$ be the closed set $\left\{z_{0}, z_{1}, \cdots\right\}$. Since the $z_{n}$ are all distinct, the rule $g\left(z_{n}\right)=w_{n}$ defines a function $g$ on $E$. We have $g(E) \subset K$. Moreover $g$ is continuous on $E$ because $w_{n} \rightarrow w_{0}$. Assume that $g$ has been extended to a function in $\bar{U}$ and apply $\Phi$ to $g$. Since $\Phi[g]$ is continuous,

$$
\left|\Phi[g]\left(z_{0}\right)-\Phi[g]\left(z_{n}\right)\right|
$$

can be made arbitrarily small by a suitable choice of $n$. Yet our construction shows that

$$
\begin{aligned}
& \left|\Phi[g]\left(z_{0}\right)-\Phi[g]\left(z_{n}\right)\right| \\
& \quad=\left|\Phi^{*}\left(z_{0}, w_{0}\right)-\Phi^{*}\left(z_{n}, w_{n}\right)\right| \\
& \quad \geqq\left|\Phi^{*}\left(z_{0}, w_{0}\right)-\Phi^{*}\left(z_{0}, w_{n}\right)\right|-\left|\Phi^{*}\left(z_{0}, w_{n}\right)-\Phi^{*}\left(z_{n}, w_{n}\right)\right| \\
& \quad>\varepsilon-\varepsilon / 2=\varepsilon / 2 .
\end{aligned}
$$

This contradiction shows that $w \rightarrow \Phi^{*}\left(z_{0}, w\right)$ is continuous when $\left|z_{0}\right|=1$. 
Suppose that $\Phi$ is not continuous at some $f_{0} \in \bar{U}$. This means there are functions $f_{n}$ in $\bar{U}$ and $\varepsilon>0$ satisfying $\left\|f_{n}-f_{0}\right\| \rightarrow 0$ and $\left\|\Phi\left[f_{n}\right]-\Phi\left[f_{0}\right]\right\| \geqq \varepsilon$. For each $n \geqq 1$ there is a $z_{n}$ in the closed unit disk such that

$$
\left|\Phi\left[f_{n}\right]\left(z_{n}\right)-\Phi\left[f_{0}\right]\left(z_{n}\right)\right| \geqq \varepsilon .
$$

By the maximum principle we can choose $z_{n}$ with $\left|z_{n}\right|=1$. By passing to a subsequence we may assume that the $z_{n}$ converge to a point $z_{0}$.

It must be that only finitely many $z_{n}$ are equal to $z_{0}$. Otherwise $w \rightarrow$ $\Phi^{*}\left(z_{0}, w\right)$ would be discontinuous at $f_{0}\left(z_{0}\right)$. (Put $w_{n}=f_{n}\left(z_{0}\right)$ in the preceding argument.) We may therefore assume that $z_{1}, z_{2}$, etc. are distinct from $z_{0}$ and, since they converge to $z_{0}$, from each other.

Let $E$ be the closed set $\left\{z_{0}, z_{1}, \cdots\right\}$ and define $g$ on $E$ by $g\left(z_{n}\right)=f_{n}\left(z_{n}\right)$. Since $f_{n} \in \bar{U}$, we have $g(E) \subset K$, and $g$ is continuous on $E$ because $\left|g\left(z_{0}\right)-g\left(z_{n}\right)\right| \leqq\left|f_{0}\left(z_{0}\right)-f_{0}\left(z_{n}\right)\right|+\left\|f_{0}-f_{n}\right\|$. Again use Rudin's extension result to extend $g$ to a function in $\bar{U}$. Apply $\Phi$ to $g$. The mapping $\Phi$ operates pointwise, so $\Phi[g]\left(z_{n}\right)=\Phi^{*}\left(z_{n}, g\left(z_{n}\right)\right)=\Phi^{*}\left(z_{n}, f_{n}\left(z_{n}\right)\right)=\Phi\left[f_{n}\right]\left(z_{n}\right)$. For $n \geqq 1$ we get

$$
\begin{aligned}
& \left|\Phi[g]\left(z_{n}\right)-\Phi[g]\left(z_{0}\right)\right| \\
& \quad=\left|\Phi\left[f_{n}\right]\left(z_{n}\right)-\Phi\left[f_{0}\right]\left(z_{0}\right)\right| \\
& \quad \geqq\left|\Phi\left[f_{n}\right]\left(z_{n}\right)-\Phi\left[f_{0}\right]\left(z_{n}\right)\right|-\left|\Phi\left[f_{0}\right]\left(z_{n}\right)-\Phi\left[f_{0}\right]\left(z_{0}\right)\right| \\
& \quad \geqq \varepsilon-\left|\Phi\left[f_{0}\right]\left(z_{n}\right)-\Phi\left[f_{0}\right]\left(z_{0}\right)\right| .
\end{aligned}
$$

This contradicts the fact that both $\Phi[g]$ and $\Phi\left[f_{0}\right]$ are continuous at $z_{0}$. Thus $\Phi$ is continuous at each $f_{0} \in \bar{U}$.

Proof of Theorem 1. It suffices to prove that if $\Phi: \bar{U} \rightarrow A$ is pointwise then $\Phi$ is $L$-analytic on $U$. For suppose this is known and suppose $\Psi$ is a pointwise mapping of an open ball $B$ into $A$. Let $B$ be an open ball of radius $r$ about $f_{0}$. Note that a composition of pointwise ( $L$-analytic) mappings is again pointwise ( $L$-analytic), and that a linear mapping $f \rightarrow c f+g$ with $c \in C$ and $g \in A$ is both pointwise and $L$-analytic. For $0<s<r$ define $\Phi_{s}: \bar{U} \rightarrow A$ by $\Phi_{s}[f]=\Psi\left[s f+f_{0}\right]$. Since $\Phi_{s}$ is pointwise on $\bar{U}, \Phi_{s}$ is $L$-analytic on $U$. For $\left\|f-f_{0}\right\|<s$, we have $\Psi[f]=\Phi_{s}\left[s^{-1}\left(f-f_{0}\right)\right]$; therefore $\Psi$ is $L$ analytic on the open ball of radius $s$ about $f_{0}$. Since $s$ can approach $r$ from below and since $L$-analyticity is a local property (as in the classical case), $\Psi$ is $L$-analytic on $B$.

From now on we assume that $\Phi: \bar{U} \rightarrow A$ is a given pointwise mapping. By Lemma $3, \Phi$ is continuous. In particular $\Phi$ maps $T \subset \bar{U}$ continuously into $A$. By Lemma 2 we define an $L$-analytic mapping $I$ on $U$ by

$$
I[f]=(2 \pi i)^{-1} \int_{T}(\zeta-f)^{-1} \Phi[\zeta] d \zeta .
$$


We shall show that $\Phi$ and $I$ agree on $U$. Since $I$ is the Cauchy integral of $\Phi$, this is what we should expect if the theorem is true.

We examine the functions $\Phi[z]$ for $z \in K$. They are important because they determine the pointwise mapping $\Phi$. For if we wish to compute $\Phi[f](w)$ for some $f \in \bar{U}$ and $w \in K$, we have

$$
\Phi[f](w)=\Phi^{*}(w, f(w))=\Phi[f(w)](w) .
$$

This makes sense because $f(w) \in K$. For $|w|<1$ we can expand $\Phi[z](w)$ in a power series

$$
\Phi[z](w)=\sum_{n=0}^{\infty} C_{n}(z) w^{n},
$$

and using this with $z=f(w)$ in (2) we get

$$
\Phi[f](w)=\sum_{n=0}^{\infty} C_{n}(f(w)) w^{n} .
$$

As the $C_{n}(z)$ appear in (3) they are simply some functions of $z$. We shall show that they are in fact continuous and then that they belong to $A$. We know $\Phi$ is continuous on $K \subset \bar{U}$. Since $K$ is compact, $\Phi$ is uniformly continuous on $K$. That is, given $\varepsilon>0$, there is a $\delta>0$ such that $z$ and $w \in K$ and $|z-w|<\delta$ imply $\|\Phi[z]-\Phi[w]\|<\varepsilon$. We can represent $C_{n}(z)$ and $C_{n}(w)$ by the classical Cauchy integral formula for derivatives. Doing this and keeping $|z-w|<\delta$, we get

$$
\begin{aligned}
\left|C_{n}(z)-C_{n}(w)\right| & =\left|(2 \pi i)^{-1} \int_{T}(\Phi[z](\zeta)-\Phi[w](\zeta)) \zeta^{-n-1} d \zeta\right| \\
& \leqq(2 \pi)^{-1} \int_{T}\|\Phi[z]-\Phi[w]\||d \zeta|<\varepsilon .
\end{aligned}
$$

This shows that $C_{n}$ is continuous on $K$. (We have actually shown that the $C_{n}$ form an equicontinuous family.)

To show the $C_{n}$ belong to $A$ we use Lemma 1 and the $A$-valued maps $\alpha, \beta, \gamma_{k}: T \rightarrow A$ given by $\alpha[\zeta](z)=\zeta^{-1} z, \beta[\zeta](z)=\Phi[\alpha[\zeta]](\zeta z)$ and $\gamma_{k}[\zeta]=$ $\zeta^{-k-1} \beta[\zeta]$. These maps are continuous. With $|z|<1$ and $w=\zeta z$ equation (4) gives

$$
\Phi[\alpha[\zeta]](w)=\sum_{n=0}^{\infty} C_{n}\left(\zeta^{-1} w\right) w^{n}
$$

and

$$
\gamma_{k}[\zeta](z)=\sum_{n=0}^{\infty} C_{n}(z) \zeta^{n-k-1} z^{n}
$$


Put $b_{k}=(2 \pi i)^{-1} \int_{T} \gamma_{k}[\zeta] d \zeta$. Lemma 1 shows that $b_{k} \in A$. For each $|z|<1$ the series (6) converges uniformly in $\zeta$, and so

$$
b_{k}(z)=\sum_{n=0}^{\infty} C_{n}(z) z^{n}(2 \pi i)^{-1} \int_{T} \zeta^{n-k-1} d \zeta=C_{k}(z) z^{k} .
$$

Since $C_{k}(z)=z^{-k} b_{k}(z)$, we know $C_{k}(z)$ is analytic for $|z|<1$ except possibly for a pole at $z=0$. But we also know that $C_{k}$ is continuous on $K$, so in fact $C_{k} \in A$.

We return to the $L$-analytic mapping $I$. Fix $f \in U$ and $|w|<1$. Using (1) and expansion (3), we get

$$
\begin{aligned}
I[f](w) & =(2 \pi i)^{-1} \int_{T}(\zeta-f(w))^{-1} \Phi[\zeta](w) d \zeta \\
& =\sum_{n=0}^{\infty} w^{n}(2 \pi i)^{-1} \int_{T}(\zeta-f(w))^{-1} C_{n}(\zeta) d \zeta .
\end{aligned}
$$

Since the $C_{n}$ belong to $A$, the above sum of Cauchy integrals gives us

$$
I[f](w)=\sum_{n=0}^{\infty} w^{n} C_{n}(f(w)) .
$$

Refer back to (4) to see that the right-hand side of (8) is $\Phi[f](w)$. Thus $I[f]$ and $\Phi[f]$ agree on the interior of $K$. Since both are continuous on $K, \Phi[f]=I[f]$. The $f$ was any function in $U$; therefore the restriction of $\Phi$ to $U$ is the $L$-analytic mapping $I$, and the theorem is proved.

Since the pointwise property implies so much in the disk algebra, it seems natural to ask what further properties it implies in other function spaces. The spaces $C(X)$ indicate some possibilities. Here $X$ stands for a compact Hausdorff space, and $C(X)$ has the topology of the uniform norm.

$L$-analyticity is too much to look for. Consider the pointwise transformation that sends each function in $C(X)$ to its complex conjugate. In view of Lemma 3 one might expect pointwise transformations to be continuous. That too is false. If $x_{0}$ is an isolated point of $X$, then we define a discontinuous pointwise transformation $\Phi$ in $C(X)$ by $\Phi^{*}(x, z)=z$ when $x \neq x_{0}$ or $z \neq 0$ and $\Phi^{*}\left(x_{0}, 0\right)=1$. Lemma 3 can be modified to give the following result.

THEOREM 2. If $X$ is a compact Hausdorff space which is first countable and has no isolated points, then every locally pointwise transformation in $C(X)$ is continuous.

Proof. Let $\Phi$ be a locally pointwise transformation in $C(X)$. We wish to show that $\Phi$ is continuous at each function in its domain. Let $U$ be the 
open unit ball in $C(X)$. By composing $\Phi$ with affine mappings of $C(X)$ into itself, we can reduce the problem to showing that $\Phi$ is continuous at the zero function when $\Phi$ is pointwise on $U$.

As in Lemma 3 some general remarks are in order. First, if $E$ is a closed subset of $X$ and if $g$ is a continuous mapping of $E$ into the open unit disk in $C$, then $g$ extends to a function in $U$. That is the Tietze extension theorem. Second if $g \in U$ then $\Phi[g]$ is continuous on $X$. Because $\Phi$ maps $U$ into $C(X)$. Third, if $x_{0} \in X$ then there is a sequence of points in $X$ which converge to $x_{0}$ and are distinct from each other and from $x_{0}$. That is because $X$ is first countable and has no isolated points.

From here the proof follows that of Lemma 3, except there is no need to choose points from a special subset such as the unit circle.

As a special case one can verify the conclusion of Theorem 2 when $X$ is the one point compactification of the long line. Therefore first countability is not a necessary condition. Precise conditions on $X$ must have to do with the existence of suitable continuous functions.

\section{REFERENCES}

1. B. W. Glickfeld, On the inverse function theorem in commutative Banach algebras, Illinois J. Math. 15 (1971), 212-221. MR 42 \#8287.

2. E. Hille, Analytic function theory. Vol. 1, Introduction to Higher Math., Ginn, Boston, Mass., 1959, pp. 182-183. MR 21 \#6415.

3. E. R. Lorch, The theory of analytic functions in normed Abelian vector rings, Trans. Amer. Math. Soc. 54 (1943), 414-425. MR 5, 100.

4. C. E. Rickart, Analytic functions of an infinite number of complex variables, Duke Math. J. 36 (1969), 581-597. MR 40 \#7819.

5. W. Rudin, Boundary values of analytic functions, Proc. Amer. Math. Soc. 7 (1956), 808-811. MR 18, 472.

6. H. E. Warren, A Riemann mapping theorem for $C(X)$, Proc. Amer. Math. Soc. 28 (1971), 147-154. MR 43 \#5300.

Department of Mathematics, University of Arizona, Tucson, Arizona 85721 